\title{
Distribution géographique des cercaires parasites des Mollusques du genre Hydrobia Hartman \\ des côtes de France
}

\author{
par S. DEBLOCK \\ Laboratoire de Parasitologie, Faculté de Pharmacie, rue du P'-Laguesse, F 59045 Lille.
}

\section{Résumé.}

L'auteur énumère les quarante-huit espèces de trématodes larvaires différentes observées de 1973 à 1977 dans 20000 mollusques des espèces $H$. ulvae, $H$. acuta et $H$. ventrosa prélevés dans quinze stations réparties en douze points du littoral (sept sur la Manche, trois sur l'Atlantique et deux sur la Méditerranée). Des tableaux énumèrent la liste des trois à vingtdeux espèces de trématodes découvertes dans les çuinze stations et résument la prévalence du parasitisme dans chaque population ainsi que, éventuellement, son incidence en fonction des années.

\section{Summary.}

Geographical distribution of the cercariae parasites of the molluses belonging to the genus Hydrobia in France.

The author lists forty eight different species of larval digenean trematodes observed in 20000 molluscs belonging to the genus Hydrobia ( $H$. ulvae, $H$. acuta and $H$. ventrosa). These hosts were examined from twelve different salt marshes and estuaries around the coasts of France (seven on the Channel, three on the Atlantic Ocean and two on the Mediterranean sea). The collections were made in fifteen sites from 1973 to 1977 . The list of the species of larval trematodes found in each site is shown in fifteen tables. These one sum up the prevalence of parasitism in each population of molluscs collected and at times its incidence over a period of two years or more.

Accepté le 6 juin 1978. 


\section{Introduction}

Les larves des trématodes parasites de 20000 mollusques du genre Hydrobia Hartman des côtes de France ont été répertoriés de 1973 à 1977 dans douze gîtes différents répartis sur les trois principales côtes maritimes de la France continentale. Une étude préliminaire (Deblock, 1978) a eu pour objet de dresser une liste provisoire des espèces, chacune de celles-ci étant accompagnée d'une description sommaire. Une étude complétée et plus détaillée, à paraître ultérieurement, concernera les quarantehuit espèces observées, leur discussion et les références bibliographiques correspondantes.

Les cercaires des Hydrobia s'enkystent dans le milieu extérieur ou pénètrent dans des crustacés, des annélides ou d'autres mollusques vivant dans leur environnement proche. Les métacercaires évoluent à l'état adulte chez des poissons marins ou des oiseaux de rivage. Cependant, le cycle évolutif de plusieurs espèces n'est pas encore connu. A des aspects morphologiques identiques ou presque identiques des cercaires correspondent parfois des espèces distinctes des trématodes adultes; ces difficultés d'interprétation expliquent certaines imprécisions de la nomenclature qui a été établie.

\section{Répartition des gîtes de prélèvement des Hydrobia}

La répartition des gîtes où furent prélevés les Hydrobia le long des côtes de France est la suivante, du Nord au Sud. $1^{\circ}$ Côtes de la Manche: embouchures de la Slack, de la Canche et de l'Authie ; estuaires de la Somme (2 stations) et de la Vire ( 2 stations). Baies de Portbail et de Lessay. $2^{\circ}$ Côtes de l'Aťlantique : île d'Oléron, baie d'Arcachon et lac d'Hossegor. $3^{\circ}$ Côtes de la Méditerranée : étangs du Canet $(2$ stations) et de l'Arnel. Il y eut au total 15 points de prélèvement. Les observations furent renouvelées dans plusieurs de ces points, mais de façon irrégulière dans le temps.

\section{Nature des hôtes}

Les gîtes océauiques (Manche, Atlantique) n'abritent qu'une seule espèce de Mollusque, Hydrobia ulvae (Pennant, 1777) (=Peringia u.). Deux espèces fréquentent simultanément les gîtes de la Méditerranée : H. acuta (Draparnaud, 1805) et H. ventrosa (Montagu, 1803) (= Paludestrina stagnalis Baster).

Tous les mollusques examinés sont des adultes âgés au moins d'un an. Les juvéniles nés dans l'année ont été épisodiquement contrôlés et se sont toujours révélés négatifs ; ils n'ont pas été comptabilisés. 
Tableau I. Nomenclature des espèces de Trématodes digènes, parasites des Hydrobia des Côtes de France.

\begin{tabular}{|c|c|c|c|c|c|c|c|c|c|}
\hline \multirow[b]{2}{*}{ GROUPE } & \multirow[b]{2}{*}{ TYPES } & \multirow[b]{2}{*}{ FAMILLES } & \multirow[b]{2}{*}{$\mathrm{N}^{-}$} & \multirow[b]{2}{*}{ DENOMINATION DE L'ESPECE } & \multicolumn{4}{|c|}{\begin{tabular}{|l|} 
HOTES \\
Hydrobia \\
\end{tabular}} & \multirow[b]{2}{*}{$\begin{array}{l}\text { PREMIERE MENTION } \\
\text { EN FRANCE }\end{array}$} \\
\hline & & & & & \multicolumn{2}{|c|}{\begin{tabular}{|l|l|l} 
ulvac \\
MandAll.
\end{tabular}} & \multicolumn{2}{|c|}{$\frac{\text { ventacu }}{\text { Med. }}$} & \\
\hline 1 & FURCOCERQUE & SCHISTOSOMATIDAE & 1 & Furcolophocercaire sp. n" 1 & & & * & $*$ & - \\
\hline " & CYSTOCERQUE & HEMIURIDAE & $\begin{array}{l}1 \\
2 \\
3\end{array}$ & $\begin{array}{l}\text { Cercaria sinitsini Rothsehild, } 1938 \\
\text { Cercaire a panache sp. } n^{-} 2 \\
\text { C. Bumorotyle progenetica (Markowski, 1936) }\end{array}$ & * & $\begin{array}{l}* \\
*\end{array}$ & * & & DEBLOCK. 1974 \\
\hline \multirow{4}{*}{ III } & \multirow{4}{*}{ LEPTOCERQUE } & HAPLOPORIDAE & \begin{tabular}{r|r}
$A$ & 1 \\
2 & \\
3 & \\
4 & \\
6 & \\
7 \\
8
\end{tabular} & 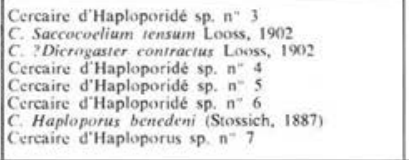 & $\begin{array}{l}* \\
* \\
* \\
*\end{array}$ & $\begin{array}{l}* \\
* \\
* \\
* \\
*\end{array}$ & * & & $\begin{array}{l}\text { FARÈS at UAILLARD. } 1974 \\
\text { FARÉS at MAILLARD. } 1974 \\
\vdots \\
\text { FARÉS et MAILLARD. } 1974 \\
\text { - }\end{array}$ \\
\hline & & HAPLOSPLANCHNIDAE & 9 & C. Haplosplanchus pachysomus (Eyscrnhardt, 1829) & & & * & & FARÈS, 1974 \\
\hline & & ACANTHOCOLPIDAE & 10) & C. Deropristis intlara (Molin, 1858) & * & * & * & * & MAILLARD, 1976 \\
\hline & & ECHINOSTOMATIDAE & $\begin{array}{r}B \\
2 \\
3 \\
4 \\
5 \\
6\end{array}$ & 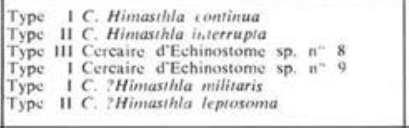 & $\begin{array}{l}* \\
* \\
* \\
* \\
* \\
* \\
*\end{array}$ & * & * & * & $\begin{array}{l}\vdots \\
\text { TIMON-DAVID at REBECQ. } 1958 \\
\text { SELLLIN, 1970 }\end{array}$ \\
\hline IV & LOPHOCERQUE & PSILOSTOMATIDAE & $\begin{array}{l}1 \\
2\end{array}$ & $\begin{array}{l}\text { C. Psilostomum hrevicolle (Creplin. 1829) } \\
\text { C. Psilorhasmus aelyptorr his Loos Frank, } 1968\end{array}$ & & * & * & & : \\
\hline $\mathrm{v}$ & MONOSTOME & NOTOCOTYLIDAE & $\begin{array}{l}1 \\
2 \\
2 \\
4 \\
3 \\
5\end{array}$ & 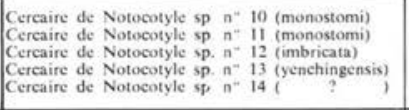 & $\begin{array}{l}* \\
* \\
*\end{array}$ & * & * & & $\vdots$ \\
\hline \multirow[t]{3}{*}{ vi } & $\begin{array}{l}\text { XIPHIDIO } \\
\text { cercairs }\end{array}$ & $\begin{array}{l}\text { leptocerque } \\
\text { monostome } \\
\text { MICROPHALLIDAE }\end{array}$ & \begin{tabular}{rr|r}
$A$ & 1 \\
2 & \\
3 & \\
4 & \\
5 & \\
6 & \\
8 & \\
9 & \\
10 & \\
11 & \\
12 & &
\end{tabular} & 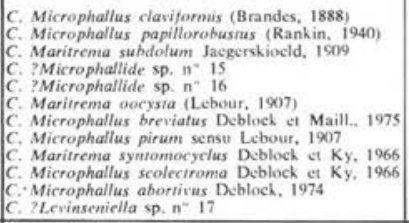 & $\begin{array}{l}* \\
* \\
* \\
* \\
* \\
*\end{array}$ & $\begin{array}{l}* \\
* \\
* \\
* \\
* \\
* \\
* \\
*\end{array}$ & $\begin{array}{l}* \\
* \\
* \\
* \\
* \\
* \\
* \\
* \\
*\end{array}$ & $\begin{array}{l}* \\
* \\
* \\
* \\
* \\
* \\
* \\
* \\
* \\
*\end{array}$ & $\begin{array}{l}\text { DEBLOCK et ROSÉ, } 1965 \\
\text { REBECQ, } 1961 \\
\text { CARRERE, } 1936 \\
: \\
\text { DEBLOCK, } 1975 \\
\text { DEBLOCK ot MAILLARD, } 1975 \\
\text { DEBLOCK \& KY, } 1966 \\
\text { DEBLOCK of KY. } 1966 \\
\text { DEBLOCK, } 1974 \\
\text { DEBLOCK, } 1974 \\
\end{array}$ \\
\hline & $X$. MICROCERQUE & & B 1 & C. ramarguensis Rebeeq, 1964 & * & * & * & * & REBECQ. 1964 \\
\hline & X BISTOME & PLAGiorCHIIDAE & $\mathrm{C} 2$ & Cercaire virgule sp. $n-18$ & & & * & & . \\
\hline \multirow[b]{3}{*}{ vII } & \multicolumn{4}{|c|}{ MONOSTOME à organe protractile de pénétration } & & & & & \\
\hline & LEPTOCERQUE & & A) 1 & Cercaire oecllée sp. $n^{n} 20$ & * & * & * & & - \\
\hline & $\begin{array}{l}\text { PLEUROLOPHO- } \\
\text { CERQUE }\end{array}$ & $\begin{array}{l}\text { HETEROPHYIDAE } \\
\text { ACANTHOSTOMATIDAE } \\
\text { CRYPTOGONIMIDAE }\end{array}$ & \begin{tabular}{|l|l|l}
$\begin{array}{l}\text { B) } \\
2 \\
3 \\
4 \\
4\end{array}$ & 0 \\
\end{tabular} & \begin{tabular}{|l|} 
C. ietralophocerra Rebceq, 1964 \\
C. Cryppocotyle iejuna (Nicoll, 1907) \\
C. Timoniella practerinum (Looss, 1901) \\
C. Aphalloides roelomicola Dollfus et coll., 1957
\end{tabular} & $\begin{array}{l}* \\
* \\
* \\
*\end{array}$ & $\begin{array}{l}* \\
* \\
*\end{array}$ & $\begin{array}{l}* \\
* \\
?\end{array}$ & * & $\begin{array}{l}\text { REBECQ, } 1964 \\
\text { MAILLARD, } 1974 \\
\text { MAILLARD, } 1974\end{array}$ \\
\hline VIII & CERCARIAEUM & GYMNOPHALLIDAE & 13 & $\begin{array}{l}\text { Mc. Gymnophallus glandosa (Lebour, 1908) } \\
\text { Mc. Gymnophallus sp. } n^{-1} \text { ( (Rebceq. 1964) }\end{array}$ & * & * & * & * & REBECQ, 1964 \\
\hline
\end{tabular}




\section{Nomenclature des cercaires de Trématodes digènes}

La nomenclature des espèces de trématodes digènes parasites des Hydrobia est présentée dans le tableau I. Les cercaires répertoriées y sont classées : $1^{\circ}$ en fonction de l'aspect morphologique, leur caractère le plus immédiatement accessible (groupes I à VIII) ; $2^{\circ}$ par famille, chaque fois que celle-ci est déterminable. La liste des cercaires est suivie de leur répartition en fonction de la nature des hôtes et de leur origine géographique (côtes de la Manche, de i'Atlantique ou de la Méditerranée). La dernière colonne du tableau fait l'historique des auteurs qui ont mentionné l'espèce pour la première fois en France. Une douzaine d'espèces n'avaient pas encore été signalées à ce jour.

La désignation spécifique des cercaires n'a été mentionnée que dans les cas où l'identification ne pose que peu de difficultés relatives, en fonction des données de la littérature française et européenne. Dans les autres cas, le parasite est désigné par un numéro d'ordre, fonction de son type morphologique, de sa famille et de sa place dans chaque liste partielle constituée (exemple: III A 1-cercaire sp. $\mathrm{n}^{\circ} 3$ ). Les descriptions détaillées des espèces, à paraître ultérieurement, porteront les mêmes références.

\section{Inventaire des cercaires dans chacun des 15 points de prélèvement}

Les parasites des Hydrobia présents dans chaque gîte et station sont énumérés dans les tableaux II à XVI qui mentionnent en outre : $1^{\circ}$ les dates de prélèvement (année et mois) ; $2^{\circ}$ le nombre de mollusques autopsiés. En regard de chaque espèce de cercaire présente dans le gîte, figure le nombre des Hydrobia porteurs du parasite. Le tableau indique enfin, éventuellement, le nombre des espèces de parasites non déterminables (cercaires absentes ou immatures) et le nombre des mollusques atteints d'une infestation multiple.

\section{A) Manche.}

Tableau II. - Gîte de la Slack (rive nord), département du Pas-de-Calais.

\begin{tabular}{|c|c|c|c|}
\hline \multirow{2}{*}{ Localisation : la Slack } & Années & 1975 & 1977 \\
\hline & Mois & 7 & 5 \\
\hline Dénomination des cercaires & $\begin{array}{c}\text { Nombre } \\
\text { d'autopsies }\end{array}$ & 300 & 300 \\
\hline & Hôtes & \multicolumn{2}{|c|}{ H. ulvae } \\
\hline II $3 \ldots \ldots \ldots . .$. Cercaria Bunocotyle progenetica & & 16 & 2 \\
\hline III A 10 ......... Cercaria Deropristis inflata & & 1 & \\
\hline III B $1 \ldots \ldots \ldots \ldots$ Cercaria Himasthla sp. type I & & 1 & \\
\hline VI A 1 ............ Cercaria Microphallus claviformis & & & 1 \\
\hline VII B 3 .......... Cercaria Timoniella praeteritum & & & 1 \\
\hline
\end{tabular}

$\mathrm{Ni}$ indéterminable ni infestation mixte 
Tableau III. - Gite de la Canche (rive nord), département du Pas-de-Calais.

\begin{tabular}{|c|c|c|c|}
\hline \multirow[t]{2}{*}{ Localisation : la Canche } & Années & 1974 & 1976 \\
\hline & Mois & 6 & 9 \\
\hline Dénomination des cercaires & $\begin{array}{c}\text { Nombre } \\
\text { d'autopsies }\end{array}$ & 300 & 500 \\
\hline & Hôtes & \multicolumn{2}{|c|}{ H. úlvae } \\
\hline II $3 \ldots \ldots \ldots . .$. Cercaria Bunocotyle progenetica & \multicolumn{3}{|c|}{13} \\
\hline III B 1 ......... Cercaria Himasthla sp. type I & & 1 & \multirow[t]{2}{*}{1} \\
\hline III B $2 \ldots \ldots \ldots \ldots$ Cercaria Himasthla sp. type II & & 1 & \\
\hline VI A $1 \ldots \ldots \ldots$...... Cercaria Microphallus claviformis & & 9 & 3 \\
\hline VI A 3 .......... Cercaria Maritrema subdolum & & 4 & 6 \\
\hline VII B $1 \ldots \ldots \ldots$ Cercaria tetralophocerca & & 2 & 1 \\
\hline VII B 2 ........... Cercaria Cryptocotyle jejuna & & 5 & 6 \\
\hline \multicolumn{4}{|c|}{$\mathrm{Ni}$ indéterminable ni infestation mixte } \\
\hline
\end{tabular}

Tableau IV. - Gite de l'Authie (rive sud), département du Pas-de-Calais.

\begin{tabular}{|c|c|c|}
\hline \multirow{2}{*}{ Localisation : Authie } & Années & 1974 \\
\hline & Mois & $6^{e}$ \\
\hline \multirow[t]{2}{*}{ Dénomination des cercaires } & $\begin{array}{c}\text { Nombre } \\
\text { d'autopsies }\end{array}$ & 300 \\
\hline & Hôte & H. ulvae \\
\hline III B $1 \ldots \ldots \ldots . .$. Cercaria Himasthla sp. type I & & 2 \\
\hline VI A 1 ........... Cercaria Microphallus claviformis & & 27 \\
\hline VI A 3 ........... Cercaria Maritrema subdolum & & 3 \\
\hline VII B I .......... Cercaria tetralophocerca & & 2 \\
\hline VII B 2 .......... Cercaria Cryptocotyle jejuna & & 7 \\
\hline \multicolumn{3}{|c|}{$\mathrm{Ni}$ indéterminable ni infestation multiple } \\
\hline
\end{tabular}

Gîte de la Somme, département de la Somme.

Deux stations ont été prospectées : la première (station IV) en bordure de la route nationale 40 A Noyelle-Le Crotoy, en amont du bassin de chasse, et la seconde (station V) au niveau de l'embouchure de la Maye, au nord du Crotoy. 
Tableau V. - Gîte de la Somme (département de la Somme) - Station IV.

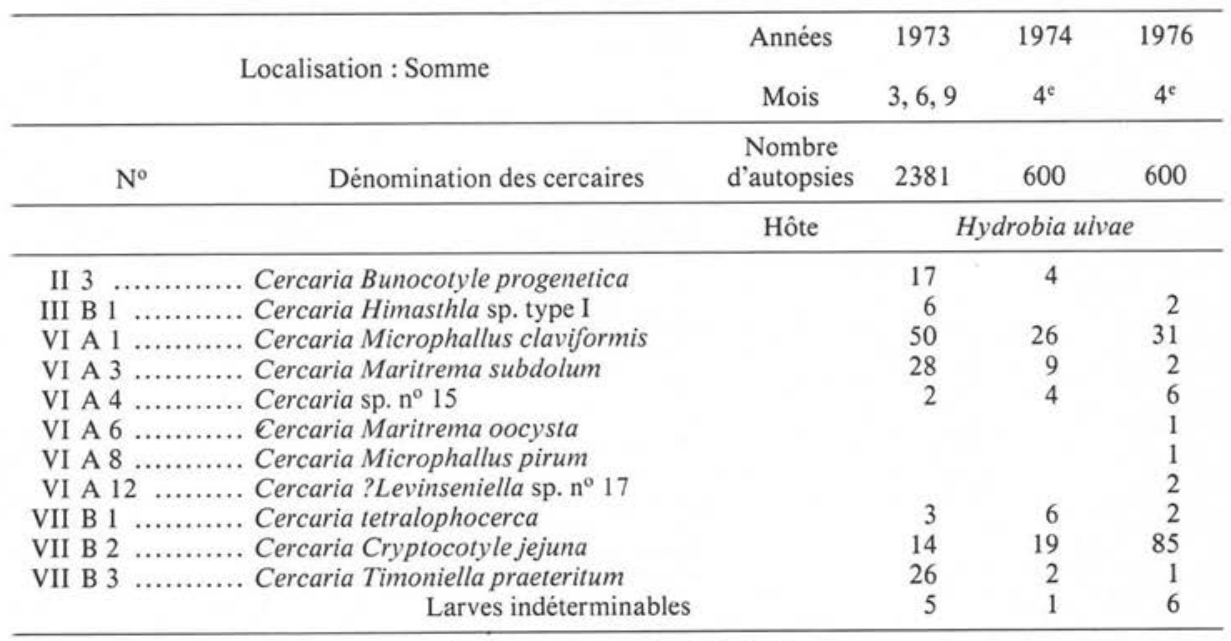

Absence d'infestation multiple

Tableau VI. - Gite de la Somme (la Maye) - Station V.

\begin{tabular}{|c|c|c|c|c|c|}
\hline \multirow{2}{*}{ Localisation : Somme (la Maye) } & Années & 1973 & 1974 & 1975 & 1976 \\
\hline & Mois & $8^{\mathrm{e}}$ & 4 et 6 & 6 et 7 & 3 et 7 \\
\hline \multirow[t]{2}{*}{ Dénomination des cercaires } & $\begin{array}{c}\text { Nombre } \\
\text { d'autopsies }\end{array}$ & 1500 & 720 & 1100 & 800 \\
\hline & Hôtes & \multicolumn{4}{|c|}{ Hydrobia ulvae } \\
\hline III A $1 \ldots \ldots \ldots$ Cercaria Saccocaelium sp. $\mathrm{n}^{\circ} 3$ & & 5 & 6 & & 1 \\
\hline III A 4 ............ Cercaria Haploporidé sp. $\mathrm{n}^{\circ} 4$ & & 4 & 2 & 1 & \\
\hline III A $8 \ldots \ldots \ldots$ Cercaria Haploporus sp. nº 7 & & & 1 & & \\
\hline III B 1 ............ Cercaria Himasthla sp. type I & & 3 & 1 & 2 & \\
\hline V . ............ Cercaires monostomes de Notocotylidés & & 3 & & 1 & 1 \\
\hline VI A 1 .......... Cercaria Microphallus claviformis & & 10 & 150 & 13 & 9 \\
\hline VI A 3 ........... Cercaria Maritrema subdolum & & 9 & 8 & 9 & 7 \\
\hline VI A $4 \ldots \ldots \ldots \ldots$ Cercaria sp. no 15 & & 15 & 4 & 8 & 2 \\
\hline VI B 1 ........... Cercaria camarguensis & . & & 1 & 1 & 10 \\
\hline VII B 1 ............. Cercaria tetralophocerca & ${ }^{\circ}$ & & 10 & 2 & 1 \\
\hline VII B 2 .......... Cercaria Cryptocotyle jejuna & & & 48 & 14 & 1 \\
\hline VII B $3 \ldots \ldots \ldots .$. Cercaria Timoniella praeteritum & & 1 & 5 & & \\
\hline VIII 1 ............. Metacercaria Gymnophallus glandosa & & + & + & & + \\
\hline Larves indéterminables & & 7 & 4 & 4 & 10 \\
\hline
\end{tabular}

Absence d'infestation multiple 
Gîte de la Vire, département de la Manche.

Deux stations ont été prospectées au niveau de la rive ouest: la première sur le banc du Grand Vey (station VI) et la seconde au lieu-dit «le port des Veys » (station VII).

Tableau VII. - Gite de la Vire, département de la Manche - Station VI.

\begin{tabular}{|c|c|c|c|c|c|}
\hline \multicolumn{2}{|r|}{ Localisation : Vire (Banc du Gd-Veys) } & Années & 1974 & 1975 & 1977 \\
\hline & & Mois & $7^{e}$ & 2 et 7 & $10^{\mathrm{e}}$ \\
\hline & Dénomination des cercaires & $\begin{array}{c}\text { Nombre } \\
\text { d'autopsies }\end{array}$ & 500 & 755 & 200 \\
\hline & & Hôtes & \multicolumn{3}{|c|}{ Hydrobia ulvae } \\
\hline II & \multicolumn{2}{|l|}{ 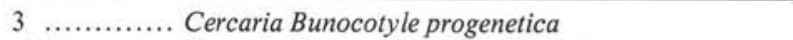 } & \multicolumn{3}{|c|}{1} \\
\hline III & A 1 .......... Cercaria Saccocaelium sp. $\mathrm{n}^{\circ} 3$ & & 18 & 8 & 6 \\
\hline III & A 4 .......... Cercaria Saccocaelium sp. $\mathrm{n}^{\circ} 4$ & & 7 & 1 & 1 \\
\hline III & A 8 .......... Cercaria Haploporus sp. $\mathrm{n}^{\circ} 7$ & & 2 & & \\
\hline III & A $10 \ldots \ldots \ldots$ Cercaria Deropristis inflata & & & 1 & \\
\hline III & B $1 \ldots \ldots \ldots .$. Cercaria Himasthla sp. type I & & 15 & 7 & \\
\hline III & B 2 .......... Cercaria Himasthla sp. type II & & 15 & 7 & 3 \\
\hline III & B 3 ......... Cercaria ?Himasthla sp. type III & & 3 & & \\
\hline \multirow[t]{2}{*}{ III } & B $4 \ldots \ldots \ldots \ldots$ Cercaria Echinostome sp. $\mathrm{n}^{\circ} 9$ & & . & . & . \\
\hline & V ........... Cercaires monostomes de Notocotylidés & & 6 & 3 & $i$ \\
\hline VI & A $1 \ldots \ldots \ldots \ldots$ Cercaria Microphallus claviformis & & 8 & 7 & 2 \\
\hline VI & A $3 \ldots \ldots \ldots .$. Cercaria Maritrema subdolum & & 6 & 17 & 1 \\
\hline VI & A 4 ........... Cercaria sp n $^{\circ} 15$ & & 7 & 12 & 1 \\
\hline VI & A $6 \ldots \ldots \ldots \ldots$ Cercaria Maritrema oocysta & & 5 & . & . \\
\hline VI & A 8 ........... Cercaria Microphallus pirum & & 2 & 1 & . \\
\hline VI & A 10 ......... Cercaria Microphallus scolectroma & & 1 & . & . \\
\hline VI & B $1 \ldots \ldots \ldots \ldots$ Cercaria camarguensis & & 1 & 1 & . \\
\hline VII & A $1 \ldots \ldots \ldots$. Cercaire ocellée monostome $n^{\circ} 20$ & & 5 & 6 & . \\
\hline VII & B $1 \ldots \ldots \ldots . .$. Cercaria tetralopphocerca & & 12 & 8 & . \\
\hline VII & В 2 .......... Cercaria Cryptocotyle jejuna & & 30 & 16 & 1 \\
\hline VII & B $3 \ldots \ldots \ldots .$. Cercaria Timoniella praeteritum & & 8 & 9 & 5 \\
\hline \multirow[t]{3}{*}{ VIII } & 1 .............. Metacercaria Gymnophallus glandosa & & + & + & + \\
\hline & Larves indéterminables & & 12 & 8 & 4 \\
\hline & Infestations multiples & & 1 & 2 & . \\
\hline
\end{tabular}


Tableau VIII. - Gîte de la Vire (Port des Veys) - Station VII.

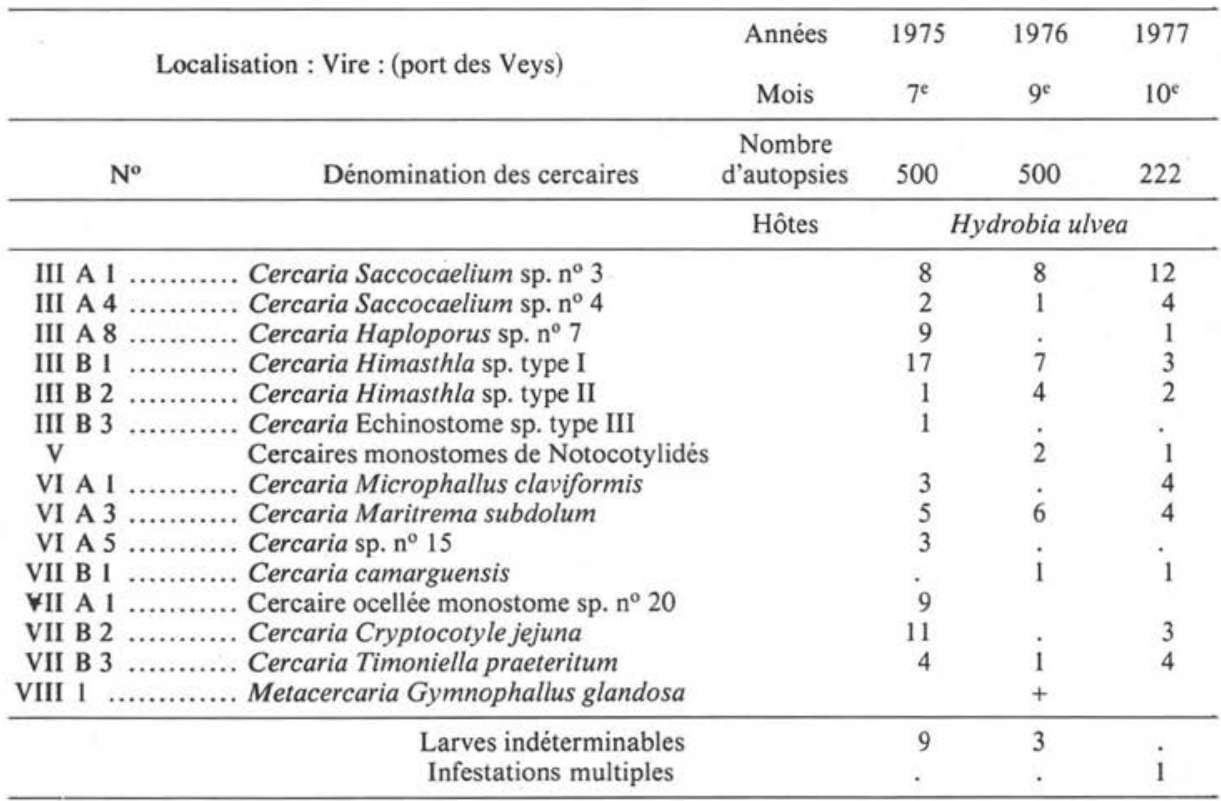

Gîtes de Portbail et de Lessay, département de la Manche.

Ces deux gîtes se situent sur la côte ouest du Cotentin.

Tableau IX. - Gite de Portbail.

\begin{tabular}{|c|c|c|}
\hline \multirow{2}{*}{ Localisation : Portbail } & Année & 1976 \\
\hline & Mois & $9^{\mathrm{e}}$ \\
\hline \multirow[t]{2}{*}{ Dénomination des cercaires } & $\begin{array}{c}\text { Nombre } \\
\text { d'autopsies }\end{array}$ & 300 \\
\hline & Hôtes & H. ulvae \\
\hline III A $1 \ldots \ldots \ldots$ Cercaria Saccocaelium $\mathrm{sp} . \mathrm{n}^{\circ} 3$ & & 1 \\
\hline III B 1 ............. Cercaria Himasthla sp. type 1 & & 1 \\
\hline V ................ Cercaire monostome de Notocotylidés & & 1 \\
\hline VI A 1 .......... Cercaria Microphallus claviformis & & 3 \\
\hline VI A 3 ........... Cercaria Maritrema subdolum & & 6 \\
\hline VII A 1 ........... Cercaire ocellée monostome sp. $\mathrm{n}^{\circ} 20$ & & 1 \\
\hline VII B 1 ........... Cercaria tetralophocerca & & 1 \\
\hline VII B 2 ............ Cercaria Cryptocotyle jejuna & & 7 \\
\hline VII B 3 ............ Cercaria Timoniella praeteritum & & 4 \\
\hline
\end{tabular}

Absence d'indéterminable et d'infestation mixte 
Tableau X. - Gite de Lessay.

\begin{tabular}{|c|c|c|c|}
\hline \multirow{2}{*}{\multicolumn{2}{|c|}{ Localisation : Lessay }} & Année & 1976 \\
\hline & & Mois & $9^{e}$ \\
\hline \multirow[t]{2}{*}{ - } & Dénomination des cercaires & $\begin{array}{c}\text { Nombre } \\
\text { d'autopsies }\end{array}$ & 300 \\
\hline & & Hôtes & H. ulvae \\
\hline \multirow{5}{*}{\multicolumn{2}{|c|}{ 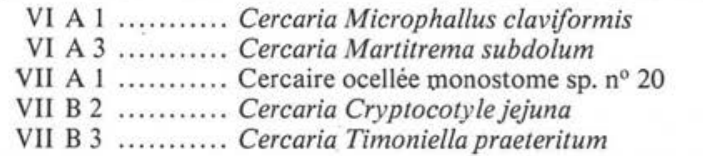 }} & & 2 \\
\hline & & & 1 \\
\hline & & & 1 \\
\hline & & & 1 \\
\hline & & & 1 \\
\hline \multicolumn{4}{|c|}{ Absence d'indéterminable et d'infestation mixte } \\
\hline
\end{tabular}

\section{B) Atlantique.}

La station se situe sur la rive Nord-est de l'île d'Oléron à l'est du chenal de Boyarville.

Tableau XI. - Gîte de l'Ile d'Oléron (département de la Charente-Maritime).

\begin{tabular}{|c|c|c|c|c|}
\hline \multirow[t]{2}{*}{ Localisation : Oléron } & Années & 1973 & 1974 & 1977 \\
\hline & Mois & $5^{\mathrm{e}}$ & $9^{e}$ & $5^{e}$ \\
\hline Dénomination des cercaires & $\begin{array}{c}\text { Nombre } \\
\text { d'autopsies }\end{array}$ & 3000 & 700 & 300 \\
\hline & Hôtes & \multicolumn{3}{|c|}{ Hydrobia ulvae } \\
\hline II 1 ............Cercaria sinitzini & & 4 & 3 & 1 \\
\hline III A 1 ............. Cercaria Saccocaelium sp. $\mathrm{n}^{\circ} 3$ & & 1 & 3 & . \\
\hline III A 4 ........... Cercaria Saccocaelium sp. $\mathrm{n}^{\circ} 4$ & & . & 3 & ${ }^{\circ}$ \\
\hline III A 8 ........... Cercaria Haploporus sp. $\mathrm{n}^{\circ} 7$ & & . & . & 1 \\
\hline III D 2 .......... Cercaria Himasthla sp. type II & & 1 & 36 & . \\
\hline III B $4 \ldots \ldots \ldots \ldots$ Cercaria Echinostome sp. n० 9 & & . & 2 & \\
\hline IV 1 ............ Cercaria Psilostomum brevicolle & & . & 3 & 2 \\
\hline * V ............... Cercaires monostomes de Notocotylidés & & & 4 & \\
\hline VI A 1 .......... Cercaria Microphallus claviformis & & 45 & 6 & 13 \\
\hline VI A 3 .......... Cercaria Maritrema subdolum & & 16 & 30 & 17 \\
\hline VI A 4 ...........Cercaria sp. $\mathrm{n}^{\circ} 15$ & & 30 & 11 & 8 \\
\hline VI A $5 \ldots \ldots \ldots \ldots$ Cercaria sp. $\mathrm{n}^{\circ} 16$ & & 1 & . & . \\
\hline VI A $8 \ldots \ldots \ldots \ldots$ Cercaria Microphallus pirum & & & 1 & . \\
\hline VI A 11 ......... Cercaria Microphallus abortivus & & 10 & 14 & . \\
\hline VI A $12 \quad \ldots \ldots \ldots$ (Cercaria) ?Levinseniella sp. $\mathrm{n}^{\circ} 17$ & & 8 & 7 & \\
\hline VI B $1 \ldots \ldots \ldots . .$. Cercaria camarguensis & & 13 & . & 2 \\
\hline VII B $2 \ldots \ldots \ldots \ldots$ Cercaria Cryptocotyle jejuna & & 13 & 14 & 1 \\
\hline VII B 3 ........... Cercaria Timoniella praeteritum & & 2 & 13 & 27 \\
\hline VIII 1 ............. Metacercaria Gymnophallus glandosa & & & + & \\
\hline Larves indeterminables & & 6 & 3 & 4 \\
\hline Infestations multiples & & . & 2 & . \\
\hline
\end{tabular}


Tableau XII. - Gîte de la baie d'Arcachon (département de la Gironde).

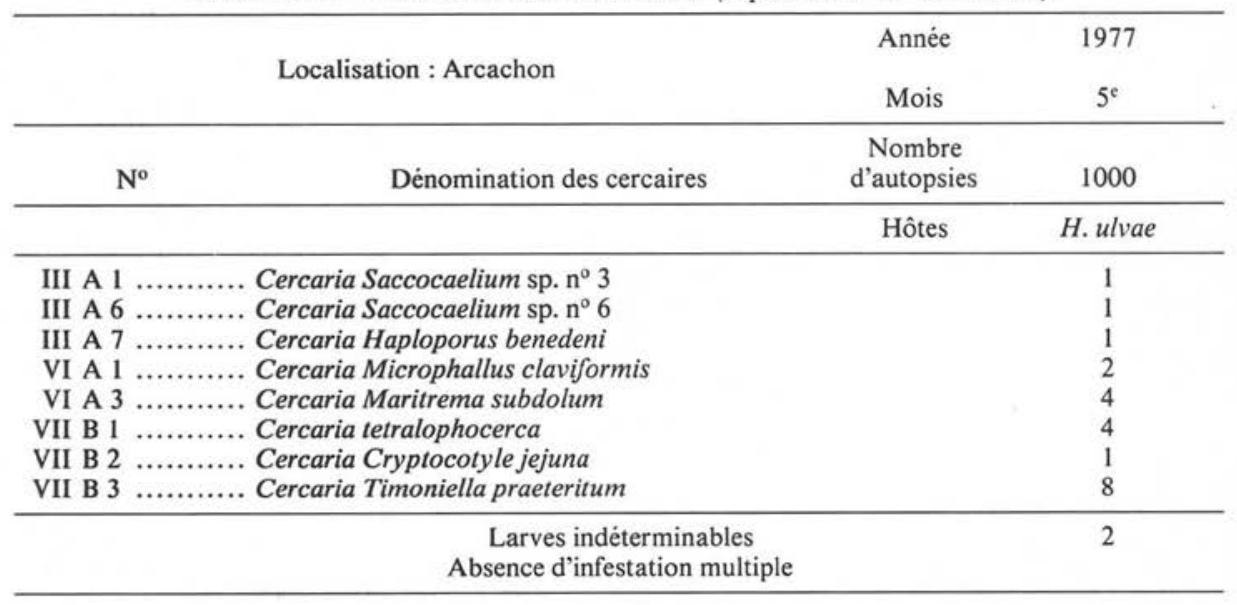

Tableau XIII. - Gîte du lac d'Hossegor (département des Pyrénées-Atlantiques).

\begin{tabular}{|c|c|c|c|}
\hline \multirow{2}{*}{\multicolumn{2}{|c|}{ Localisation : Hossegor }} & Année & 1976 \\
\hline & & Mois & $9^{e}$ \\
\hline \multirow[t]{2}{*}{$\mathrm{N}^{\mathrm{o}}$} & Dénomination des cercaires & $\begin{array}{c}\text { Nombre } \\
\text { d'autopsies }\end{array}$ & 500 \\
\hline & & Hôtes & H. ulvae \\
\hline \multirow{3}{*}{\multicolumn{3}{|c|}{$\begin{array}{l}\text { III .............. Bunocotyle progenetica } \\
\text { VI A } 3 \ldots \ldots \ldots \text { Cercaria Maritrema subdolum } \\
\text { VII A } 1 \ldots \ldots \ldots \ldots \text {. Cercaria ocellée monostome sp. } \mathrm{n}^{\circ} 20\end{array}$}} & 26 \\
\hline & & & 1 \\
\hline & & & 1 \\
\hline \multicolumn{4}{|c|}{ Absence d'indéterminable et d'infestation multiple } \\
\hline
\end{tabular}

\section{C) Méditerranée.}

Deux stations ont été prospectées dans l'étang du Canet; la première sur la rive sud (station XIII) et la seconde sur la rive est (station XIV). 
Tableau XIV. - Gîte de l'étang du Canet (département des Pyrénées Orientales) - Station XIII

\begin{tabular}{|c|c|c|}
\hline \multirow{2}{*}{ Localisation : le Canet (sud) } & Annèe & 1977 \\
\hline & Mois & $9^{e}$ \\
\hline Dénomination des cercaires & $\begin{array}{c}\text { Nombre } \\
\text { d'autopsies }\end{array}$ & 700 \\
\hline & Hôtes & H. ventrosa \\
\hline I 1 ............ Furcocercaire sp. $\mathrm{n}^{\circ} 1$ & & 4 \\
\hline III A $2 \ldots . . \ldots \ldots$ Cercaria Saccocaelium tensum & & 135 \\
\hline III A 3 .......... Cercaria ?Dicrogaster contractus & & 3 \\
\hline III A 9 ........... Cercaria Haplosplanchnus pachysomus & & 4 \\
\hline III B $1 \ldots \ldots \ldots$. Cercaria Himasthla sp. type I & & 30 \\
\hline IV 2 ........... Cercaria Psilochasmus aglyptorchis & & 5 \\
\hline V ..................... Cercaria Notocotylidé sp. $\mathrm{n}^{\circ} 11$ & & 11 \\
\hline VI A $2 \ldots \ldots \ldots .$. Cercaria Microphallus papillorobustus & & 96 \\
\hline VI A 3 ........... Cercaria Maritrema subdolum & & 49 \\
\hline VI A 4 .............. Cercaria sp. $\mathrm{n}^{\circ} 15$ & & 1 \\
\hline VI A $7 \ldots \ldots \ldots$ Cercaria Microphallus breviatus & & 26 \\
\hline VI A $8 \ldots \ldots \ldots \ldots$ Cercaria Microphallus pirum & & 4 \\
\hline VI A 9 .......... Cercaria Maritrema syntomocyclus & & 8 \\
\hline VI A 11 ........ Cercaria Microphallus abortivus & & 3 \\
\hline VI C 1 .......... Cercaire virgule de Plagiorchiidé ${ }^{\circ} 19$ & & 1 \\
\hline VII B 3 ........... Cercaria Timoniella praeteritum & & 16 \\
\hline Larves indeterminables & & 22 \\
\hline Infestations multiples & & 28 \\
\hline
\end{tabular}

Tableau XV. - Gite de l'étang du Canet (est) - Station XIV.

\begin{tabular}{|c|c|c|c|}
\hline \multirow{2}{*}{ Localisation : le Canet (est) } & Années & 1977 & 1977 \\
\hline & Mois & $9^{\mathrm{e}}$ & $9^{e}$ \\
\hline Dénomination des cercaires & $\begin{array}{c}\text { Nombre } \\
\text { d'autopsies }\end{array}$ & 250 & 250 \\
\hline & Hôtes & acuta & ventrosa \\
\hline III A $10 \ldots \ldots \ldots$ Cercaria Deropristis inflata & & 2 & . \\
\hline III B $1 \ldots \ldots \ldots \ldots$ Cercaria Himasthla sp. type I & & 1 & . \\
\hline VI A 2 .......... Cercaria Microphallus papillorobustus & & 4 & 20 \\
\hline VI A $3 \ldots \ldots \ldots \ldots$ Cercaria Maritrema subdolum & & 11 & 5 \\
\hline VI A $7 \ldots \ldots \ldots$ Cercaria Microphallus breviatus & & 1 & 28 \\
\hline VI A 9 .......... Cercaria Maritrema syntomocyclus & & 1 & 4 \\
\hline VI A $11 \ldots \ldots \ldots$ Cercaria Microphallus abortivus & & 1 & 1 \\
\hline VII B 3 ........... Cercaria Timoniella praeteritum & & 3 & 4 \\
\hline VIII 2 ............ Metacercaria Gymnophallus sp. $\mathrm{n}^{\circ} 1$ & & 5 & 2 \\
\hline $\begin{array}{c}\text { Larves indéterminables } \\
\text { Absence d'infestation multiples }\end{array}$ & & 2 & 5 \\
\hline
\end{tabular}


Tableau XVI. - Gite de l'étang de l'Arnel (département de l'Hérault).

\begin{tabular}{|c|c|c|c|c|}
\hline \multirow{2}{*}{ Localisation : Arnel } & Années & 1975 & 1976 & 1976 \\
\hline & Mois & $1^{\text {er }}$ & $4^{e}$ & $4^{e}$ \\
\hline Dénomination des cercaires & $\begin{array}{c}\text { Nombre } \\
\text { d'autopsies }\end{array}$ & 254 & 1078 & 18 \\
\hline & Hôtes & \multicolumn{2}{|c|}{ H. ventrosa } & acuta \\
\hline I 1 ........... Furcocercaria sp. $\mathrm{n}^{\circ} 1$ & & & 1 & . \\
\hline II $\quad 2 \quad$ Cercaire cystophore a panache $\mathrm{sp} . \mathrm{n}^{\circ} 2$ & & 2 & 2 & . \\
\hline III A 2 .......... Cercaria Saccocaelium tensum & & 12 & 19 & . \\
\hline III A $3 \ldots \ldots \ldots \ldots$ Cercaria ?Dicrogaster contractus & & . & 1 & . \\
\hline III A 9 ........... Cercaria Haplosplanchnus pachysomus & & 3 & 4 & . \\
\hline III A'10 ........ Cercaria Deropristis inflata & & 1 & & . \\
\hline III B $1 \ldots \ldots \ldots \ldots$ Cercaria Himasthla sp.type I & & . & 2 & . \\
\hline VI A 2 .......... Cercaria Microphallus papillorobustus & & 13 & 18 & 1 \\
\hline VI A $3 \ldots \ldots \ldots \ldots$ Cercaria Maritrema subdolum & & 7 & 12 & 2 \\
\hline VI A $7 \ldots \ldots \ldots$ Cercaria Microphallus breviatus & & 10 & 20 & 1 \\
\hline VI A 8 ........... Cercaria Microphallus pirum & & 7 & 11 & $i$ \\
\hline VI A $11 \ldots \ldots \ldots$ Cercaria Microphallus abortivus & & . & 1 & . \\
\hline VI B 1 ........... Cercaria camarguensis & & 1 & 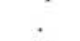 & 2 \\
\hline VII A $1 \ldots \ldots \ldots \ldots$ Cercaire ocellèe monostome sp. $\mathrm{n}^{\circ} 20$ & & 1 & 1 & . \\
\hline VII B 2 .......... Cercaria Cryptocotyle jejuna & & 2 & & . \\
\hline VII B $3 \ldots \ldots \ldots \ldots$ Cercaria Timoniella praeteritum & & 2 & 3 & . \\
\hline VII B $4 \ldots \ldots \ldots \ldots$ Cercaria Aphalloides caelomicola & & . & 1(?) & . \\
\hline VIII 2 .............. Metacercaria Gymnophallus sp. $\mathrm{n}^{\circ} 1$ & & 8 & 6 & . \\
\hline Larves indéterminables & & 7 & 15 & . \\
\hline Infestations multiples & & 2 & 2 & . \\
\hline
\end{tabular}

\section{Les infestations multiples}

Quelques mollısques ont montré des infestations par deux types de larves de trématodes.

A) Ces cas sont rares chez les hôtes des côtes océaniques. Les six cas observés se répartissent ainsi : $1^{\circ}$ dans la station VI de la Vire (tableau VII), Microphallus claviformis s'associe à une espèce non déterminée de Notocotylidé d'une part, et d'autre part à Deropristis inflata; Microphallus pirum cohabite avec des métacercaires libres de Gymnophallus glandosa; $2^{\circ}$ dans la station VII de la Vire (tableau VIII), Microphallus claviformis s'associe à Haploporus sp. $\mathrm{n}^{\circ} 7 ; 3^{\circ}$ dans le gîte d'Oléron (tableau $X I$ ), Maritrema subdolum s'associe une fois à Microphallus claviformis, et une fois à Levinseniella sp. $\mathrm{n}^{\circ} 17$.

B) Les infestations multiples sont plus fréquentes dans les gîtes de la Méditerranée. $1^{\circ}$ Cas des Hydrobia ventrosa de l'étang du Canet (tableau XIV). Saccocaelium tensum s'associe à Microphallus papillorobustus (3 fois), à $M$. abortivus ( 2 fois) et à $M$. breviatus ( 3 fois); à Maritrema subdolum (2 fois), et à $M$. syntomocyclus ( 5 fois). Haplosplanchnus pachysomus s'associe à $M$. papillorobustus (1 fois). Une cercaire 
d'Echinostome de type I s'associe à $M$. papillorobustus, à $M$. breviatus et à Maritrema syntomocyclus (chacun 1 fois), et à Microphallus abortivus (2 fois). Une cercaire de Notocotylidé s'associe à un Microphallidé sp. à cycle abrégé (1 fois). Maritrema subdolum s'associe à Maritrema syntomocyclus (2 fois), et à un Microphallidé à cycle abrégé non déterminé (1 fois). Microphallus abortivus s'associe à Timoniella praeteritum (1 fois). Microphallus papillorobustus s'associe à un Microphallidé sp. à cycle abrégé (1 fois).

$2^{\circ}$ Cas des Hydrobia ventrosa de l'étang de l'Arnel (tableau XVI).

Microphallus papillorobustus s'associe à Maritrema subdolum (1 fois), et à $M$. subdolum joint à Cercaria $\mathrm{n}^{\circ} 21$ (Cryptocotyle jejuna) 1 fois ; c'est notre seul exemple d'infestation triple. Microphallus pirum s'associe à Saccocaelium tensum (1 fois), et à une espèce indéterminable (1 fois) (1).

\section{Commentaires}

Le nombre des mollusques parasités en fonction des espèces de trématodes répertoriées ne trace qu'un tableau approché de la próvalence exacte des parasites des Hydrobia des côtes de France. Il est probable en effet que des espèces de morphologie voisine ont été confondues, au moins occasionnellement, en dépit de l'attention de l'auteur. L'étude comparée des chétotaxies aurait peut-être apporté une discrimination satisfaisante des espèces morphologiquement identiques mais elle n'a pas été entreprise ; la méthode est inadaptée au type de l'enquête menée car, si elle apporte une identification indiscutable de la cercaire du mollusque ayant fait l'objet de la technique, elle n'en apporte aucune en ce qui concerne les cercaires analogues des autres mollusques du même gîte n'ayant subi aucune préparation.

Plusieurs groupes sont difficiles à systématiser, comme par exemple celui des cercaires monostomes des Notocotylidés; l'incidence des diverses espèces du groupe est relativement faible en France, et les cercaires mûres sont d'observation difficile du fait de leur forte opacité naturelle. En conséquence, ces cercaires ont été comptabilisé globalement dans chaque gîte.

La colonne « hôtes » du tableau I résume la répartition des espèces de cercaires parasites des Hydrobia des trois rivages des mers baignant les côtes de France (Manche, Atlantique et Méditerranée). Dans les limites de notre enquête, sur la quarantaine d'espèces en liste, une douzaine apparaissent comme ubiquistes, en ce sens que cellesci se retrouvent indifféremment dans presque tous les gîtes; une vingtaine apparaissent

(1) Certaines de ces infestations mixtes sont susceptibles d'entraîner une erreur d'interprétation de la biologie des parasites associés, notamment lorsque des kystes hépato-pancréatiques d'une espèce de Microphallidé à cycle évolutif abrégé évoluent côte à côte avec les sporocytes d'une espèce à cycle évolutif à trois hôtes, telle que $M$. papillorobustus ou $M$. subdolum. Les abondantes cercaires de ces dernières espèces ne représentent pas les stades évolutifs antérieurs de la première, contrairement aux apparences. 
comme océaniques (Manche, Atlantique) ; une douzaine d'autres enfin sont méditerranéennes.

La plupart des espèces mentionnées semble endémiques, même si leur incidence varie notablement en fonction des lieux, de l'année de prélèvement et de l'espèce considérée.

Les fluctuations des incidences observées au niveau des mollusques des divers gîtes résultent vraisemblablement des hasards des passages temporaires des hôtes définitifs vertébrés (poissons ou oiseaux, selon les cas), liés aux migrations saisonnières. Ces vertébrés constituent les distributeurs épisodiques ou semi-permanents des formes infestantes des parasites offertes à la prédation du maillon stable et permanent du cycle représenté par les populations des Hydrobia sédentaires, abondantes et toujours réceptrices. Ces dernières constituent le marqueur assez fidèle d'une faune de cinquante trématodes environ actuellement établis en Europe occidentale mais qui n'ont pas tous été retrouvés chez leurs hôtes définitifs, ou même encore partiellement inconnus.

La fréquence des infestations mixtes des mollusques dans certains gîtes privilégiés tendrait à montrer que, lorsque des conditions écologiques locales favorables multiplient les occasions de rencontre hôte-parasite, le mollusque n'offre pas de résistance naturelle ou acquise à la surinfestation. Il resterait néanmoins à déterminer les modalités de la cohabitation en fonction du temps, car les deux espèces en concurrence se révèlent rarement aussi prospères l'une que l'autre.

La prévalence du parasitisme est globalement voisine de $12 \%$ sur les 20000 mollusques autopsiés ; mais elle s'abaisse à $9,8 \%$ chez $H$. ulvae des côtes océaniques, pour atteindre $27 \%$ chez les Hydrobia des gîtes méditerranéens. Cette différence du triple s'explique peut-être par la nature très différente des gîtes: vastes, très ouverts et soumis à l'alternance journalière des marées dans le premier cas ; exigüs, fermés et perpétuellement immergés dans le second, réalisant dans la nature des conditions biologiques presque semblables à celles d'un aquarium expérimental.

\section{Fréquence du parasitisme des Hydrobia}

Le classement des espèces de parasites en fonction de leur fréquence décroissante chez les Hydrobia au cours de la période 1973-1977 fournit les listes suivantes (tableau $X V I I)$.

A) Gîtes océaniques confondus (Manche et Atlantique).

$1^{\circ}$ Espèces communes (de 2,5 à $0,5 \%$ ):

Microphallus claviformis $(2,35)$. Cryptocotyle jejuna $(1,65)$. Maritrema subdolum $(1,1)$. Timoniella praeteritum $(0,67)$. Xiphidio-cercaire sp. $\mathrm{n}^{\circ} 15$ (VI A 4) $(0,62)$.

$2^{\circ}$ Espèces relativement rares (de 0,5 à $\left.0,20 \%\right)$ :

Bunocotyle progenetica $(0,43)$. Saccocaelium III A 1 sp. $\mathrm{n}^{\circ} 3(0,43)$. Himasthla interrupta, type II $(0,40)$. Cercaria tetralophocerca $(0,30)$. Himasthla continua, type I $(0,30)$. 
Tableau XVII. Nombres et pourcentages des mollusques parasités

\begin{tabular}{|c|c|c|c|c|c|c|c|c|}
\hline \multirow{4}{*}{ GROUPE } & \multirow{4}{*}{ TYPES } & \multirow{4}{*}{ FAMILLES } & \multirow{4}{*}{$\mathrm{N}^{-}$} & \multirow{4}{*}{ DENOMINATION DE L'ESPECE } & \multicolumn{4}{|c|}{ GITES } \\
\hline & & & & & \multicolumn{2}{|c|}{ octeaniques } & \multicolumn{2}{|c|}{ méditerranéens } \\
\hline & & & & & 18178 & $\%$ & 2550 & $\%$ \\
\hline & & & & & 1782 & 9,90 & 691 & 27,09 \\
\hline $\mathbf{I}$ & FURCOCERQUE & SCHISIOSOMATIDAE & 1 & Furcolophocercaire sp. n" 1 & & & 5 & 0,19 \\
\hline II & CYSTOCERQUE & HEMIURIDAE & $\begin{array}{l}1 \\
2 \\
3\end{array}$ & $\begin{array}{l}\text { Cercaria sinitzini Rothsehild, } 1938 \\
\text { Cercaire à panache sp. n" } 2 \\
\text { C. Bunocotyle progenetica (Markowski, 1936) }\end{array}$ & $\begin{array}{r}8 \\
79\end{array}$ & $\begin{array}{r}0,044 \\
0,43\end{array}$ & 4 & 0,15 \\
\hline \multirow{4}{*}{ III } & \multirow{4}{*}{ LEPTOCERQUE } & HAPLOPORIDAE & \begin{tabular}{l|l|} 
A & 1 \\
2 & \\
3 & \\
4 & \\
5 & \\
6 & \\
7 & \\
8 &
\end{tabular} & $\begin{array}{l}\text { Cercaire d'Haploporidé sp. n" } 3 \\
\text { C. Saccocoelium tensum Looss, } 1902 \\
\text { C. ?Dicrogaster contractus Looss, } 1902 \\
\text { Cercaire d'Haploporidé sp. n" } 4 \\
\text { Cercaire d'Haploporidé sp. n" } 5 \\
\text { Cercaire d'Haploporidé sp. n" } 6 \\
\text { C. Haploporus benedeni (Stossich, 1887) } \\
\text { Cercaire d'Haploporus sp. n" } 7\end{array}$ & $\begin{array}{c}78 \\
26 \\
1 \\
1 \\
14\end{array}$ & $\begin{array}{l}0,43 \\
0,14 \\
0,005 \\
0,005 \\
0,077\end{array}$ & $\begin{array}{c}166 \\
4\end{array}$ & $\begin{array}{l}6,5 \\
0,15\end{array}$ \\
\hline & & HAPLOSPLANCHNIDAE & 9 & C. Haplosplanchus pachysomus (Eysenhardt, 1829) & & & 11 & 9,43 \\
\hline & & ACANTHOCOLPIDAE & 10 & C. Deropristis inflara (Molin, 1858) & 2 & 0,01 & 3 & 0,11 \\
\hline & & ECHINOSTOMATIDAE & \begin{tabular}{l|l|}
$B$ & 1 \\
2 & \\
3 & \\
4 & \\
5 & \\
6 &
\end{tabular} & $\begin{array}{l}\text { Type I C. Himasthla continua } \\
\text { Type II C. Himasthla interrupta } \\
\text { Type III Cercaire d'Echinostome sp. } \text { n }^{*} 8 \\
\text { Type I Cercaire d'Echinostome sp. } n^{*} 9 \\
\text { Type I C. PHimasthla militaris } \\
\text { Type II C. ?Himasthla leptosoma }\end{array}$ & $\begin{array}{l}57 \\
72 \\
6 \\
2\end{array}$ & $\begin{array}{l}0,31 \\
0,40 \\
0,03 \\
0,01\end{array}$ & 33 & 1,29 \\
\hline IV & LOPHOCERQUE & PSILOSTOMATIDAE & $\begin{array}{l}1 \\
2\end{array}$ & $\begin{array}{l}\text { C. Psilostomum brevicolle (Creplin, 1829) } \\
\text { C. Psilochasmus aglyptorchis Loos Frank, } 1968\end{array}$ & 5 & 0,027 & 5 & 0,19 \\
\hline v & MONOSTOME & NOTOCOTYLIDAE & & Toutes espèces confondues & 23 & 0,12 & 11 & 0,43 \\
\hline \multirow[t]{3}{*}{ VI } & $\begin{array}{l}\text { XIPHIDIO } \\
\text { cercaire }\end{array}$ & $\begin{array}{l}\text { leptocerque } \\
\text { monostome } \\
\text { MICROPHALLIDAE }\end{array}$ & \begin{tabular}{|l|l|} 
& 1 \\
2 & \\
3 & \\
4 & \\
5 & \\
6 & \\
7 & \\
8 & \\
9 & \\
10 & \\
11 & \\
12 & \\
\end{tabular} & 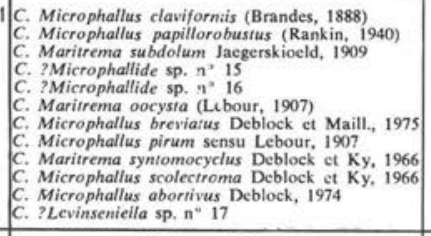 & $\begin{array}{c}424 \\
199 \\
113 \\
1 \\
6 \\
5 \\
1 \\
24 \\
17 \\
\end{array}$ & $\begin{array}{l}2,35 \\
1,1 \\
0,62 \\
0,005 \\
0,03 \\
0,027 \\
0,005 \\
0,13 \\
0,09 \\
\end{array}$ & $\begin{array}{r}152 \\
86 \\
1 \\
\\
86 \\
23 \\
13 \\
6\end{array}$ & $\begin{array}{l}5,96 \\
3,37 \\
0,03 \\
\\
3,37 \\
0,90 \\
0,50 \\
0,23\end{array}$ \\
\hline & X. MICROCERQUE & & B 1 & C. camarguensis Rebecq, 1964 & 31 & 0,17 & 3 & 0,11 \\
\hline & X. BISTOME & PLAGIORCHIIDAE & C 2 & Cercaire virgule sp. $\mathrm{n}^{\bullet} 18$ & & & 1 & 0,03 \\
\hline \multirow{2}{*}{ VII } & \multicolumn{4}{|c|}{ MONOSTOME ả organe protractile de pénétration } & 23 & 0,12 & 2 & 0,07 \\
\hline & $\begin{array}{l}\text { PLEUROLOPHO- } \\
\text { CERQUE }\end{array}$ & $\begin{array}{l}\text { HETEROPHYIDAE } \\
\text { ACANTHOSTOMATIDAE } \\
\text { CRYPTOGONIMIDAE }\end{array}$ & \begin{tabular}{l|l} 
B) 1 \\
2 \\
3 \\
4
\end{tabular} & $\begin{array}{l}\text { C. teiralophocerca Rebecq, } 1964 \\
\text { C. ?Cryptocotyle jejuna (Nicoll, 1907) } \\
\text { C. Timoniella practeritum (Looss, 1901) } \\
\text { C. A phalloides coelomicola Dollius et coll., } 1957\end{array}$ & $\begin{array}{r}54 \\
297 \\
122\end{array}$ & $\begin{array}{l}0,3 \\
1,65 \\
0,67\end{array}$ & $\stackrel{2}{28}$ & 0,07 \\
\hline VIII & CERCARIAEUM & GYMNOPHALLIDAE & $\begin{array}{l}1 \\
2 \\
\end{array}$ & $\begin{array}{l}\text { Mc. Gymnophallus glandosa (Lebour, 1908) } \\
\text { Mc. Gymnophallus sp. n" } 1 \text { (Rebecq, 1964) }\end{array}$ & + & ? & 21 & 0,82 \\
\hline
\end{tabular}


$3^{\circ}$ Espèces rares (de 0,17 à $\left.0,05 \%\right)$ :

Cercaria camarguer:sis $(0,17)$. Saccocaelium III A 4 sp. $\mathrm{n}^{\circ} 4(0,14)$. Microphallus abortivus $(0,12)$. Cercaire ocellée VII A 1 sp. $\mathrm{n}^{\circ} 20(0,12)$. Cercaria ?Levinseniella VI A 12 sp. $\mathrm{n}^{\circ} 17(0,09)$ et Haploporus III A 8 sp. $\mathrm{n}^{\circ} 7(0,07)$.

$4^{\circ}$ Espèces très rares (de 0,05 à $\left.0,01 \%\right)$ :

Cercaria sinitzini $(0,04)$. Maritrema oocysta $(0,03)$. Himasthla III B 3 sp. $\mathrm{n}^{\circ} 8$ (type III) $(0,03)$. Microphallus pirum $\left(^{*}\right)(0,027)$. Psilochasmus brevicolle $(0,02)$. Deropristis inflata $(0,01)$. Himasthla III B 4 sp. $\mathrm{n}^{\circ} 9(0,01)$.

$5^{\circ}$ Espèces exceptionnelles (en dessous de 0,01\%):

Haploporidé III A 6 sp. $\mathrm{n}^{\circ}$ 6, Haploporus benedeni $(0,005)$. Xiphidio-cercaire VI A 5 sp. $\mathrm{n}^{\circ} 16$ et Microphallus scolectroma $(0,005)$.

\section{B) Gîtes méditerranéens.}

Le même barème appliqué aux gîtes méditerranéens fournit la liste des fréquences suivantes :

$1^{\circ}$ Espèces communes (6 à $\left.0,5 \%\right)$ :

Saccocaelium tensum (6,5). Microphallus papillorobustus (5,96). Maritrema subdolum et Microphallus breviatus $(3,37)$. Himasthla continua III B I type $1(1,29)$. Timoniella praeteritum $(1,09)$. Microphallus pirum $(0,9)\left({ }^{*}\right)$. Maritrema syntomocyclus $(0,5)$.

$2^{\circ}$ Espèces relativement rares $(0,5$ à $0,05 \%)$ :

Haplosplanchnus pachysomus et cercaires de Notocotylidés $(0,43)$. Microphallus abortivus $(0,23)$.

$3^{\circ}$ Espèces rares (de 0,2 à $0,05 \%$ ):

Furcocercaire I 1 sp. $\mathrm{n}^{\circ} 1$ et Psilochasmus aglyptorchis $(0,19)$. Cercaire cystophore à panache II 2 et Dicrogaster contractus $(0,15)$. Cercaria camarguensis et Deropristis inflata $(0,11)$. Cryptocotyle jejuna et Cercaire ocellé VII A $1 \mathrm{n}^{\circ} 20(0,07)$.

$4^{\circ}$ Epèce très rare (de 0,05 à $0,01 \%$ ):

Xiphidio-cercaire VI A 5 sp. $\mathrm{n}^{\circ} 15(0,03)$.

\section{Conclusions}

L'enquête précédente est la première qui ait été menée d'une taçon systématique sur la nature et la prévalence des populations de trématodes parasites des mollusques

(*) sensu Lebour, 1907 nec Belopolskaia, 1952 et 1963. 
Hydrobia fréquentant les gîtes d'eau saumâtre des côtes de France ; à titre d'exemple, onze espèces de cercaires avaient été mentionnées par Rebecq, 1964 chez un nombre relativement restreint d'Hydrobia de Camargue (Bouches-du-Rhône). Plusieurs des espèces répertoriées en France au cours de l'enquête font naturellement partie du catalogue européen qui pourrait être dressé à partir de travaux épars, dont les plus documentés sont constitués d'une part par la série de recherches essentiellement qualitatives de Lebour (à partir de 1911) et celles de Rothschild (à partir de 1935) réalisées en Grande-Bretagne ; d'autre part, par les recherches qualitatives et quantitatives de Reimer en R.D.A. sur les rivages de la mer Baltique, à partir de 1961 ; et enfin par certains travaux expérimentaux de qualité mais plus ponctuels (Loos Frank, Maillard...). «L'inventaire des trématodes larvaires parasites des Hydrobia des côtes de France * (Deblock, à paraître) fera le point, à partir de descriptions morphologiques détaillées, des plus probables synonymies des diverses espèces cercariennes décrites en Europe chez les Hydrobia et des connaissances souvent encore fragmentaires de leur biologie.

\section{Bibliographie}

Deblock S., 1978: Les larves de trématodes parasites des Hydrobia (Mollusques prosobranches) des côtes de France. Etude préliminaire: nomenclature des espèces. Haliotis (sous presse).

Deblock S. (à paraître): Inventaire des trématodes larvaires parasites des Hydrobia (Prosobranches) des côtes de France.

Rebecq J., 1964: Recherches systématiques, biologiques et écologiques sur les formes larvaires de quelques trématodes de Camargue. Thèse Doctorat Sci. Natur., Aix-Marseille, 223 p., 15 pl. 